\title{
REFLECTIONS ON THE REPRESENTATION OF WOMEN IN THE INTERNATIONAL CONFERENCES ON AUDITORY DISPLAYS (ICAD)
}

\author{
Areti Andreopoulou
}

\author{
Laboratory of Music Acoustics and Technology \\ (LabMAT) \\ University of Athens, Greece \\ areti.andreopoulou@gmail.com
}

\author{
Visda Goudarzi \\ Institute of Electronic Music and Acoustics \\ (IEM) \\ Graz, Austria \\ goudarzieiem.at
}

\begin{abstract}
This paper investigates the representation of women researchers and artists in the conferences of the International Community for Auditory Display (ICAD). In the absence of an organized membership mechanism and / or publicly available records of conference attendees, this topic was approached through the study of publication and authorship patterns of female researchers in ICAD conferences. Temporal analysis showed that, even though there has been an increase in the number of publications co-authored by female researchers, the annual percentage of female authors remained in relatively unchanged levels (mean $=17.9 \%$ ) throughout the history of ICAD conferences. This level, even though low, remains within the reported percentages of female representation in other communities with related disciplines, such as the International Computer Music Association (ICMA) and the Conferences of the International Society for Music Information Retrieval (ISMIR), and significantly higher than in more audio engineering-related communities, such as the Audio Engineering Society (AES).
\end{abstract}

\section{INTRODUCTION}

The International Community for Auditory Display (ICAD) is a highly interdisciplinary body focusing on the use of sound to perceptualize data. The foundations of this scientific discipline were laid during the first ICAD conference in 1992, organized by Gregory Krammer [1]. Since then, ICAD has been known for bringing together researchers from various research fields, including but not limited to music technology, acoustics, psycho-acoustics, music composition, computer science, and electrical engineering.

Despite the community's strong ties to areas related to the arts and science, ICAD-related research has been dominated by male researchers. This fact can be attributed to its equally strong ties to audio engineering and other technical fields. In response to this observation, over the past few years, ICAD conference organizers have started hosting women-related events, during which such issues are openly acknowledged and discussed. However, a more systematic approach, which would promote and encourage the involvement of female researchers in leadership roles, such as session, conference, and program chairs, reviewers and metareviewers, mentors, and Board of Directors members, is still miss-

(c) (1) () (5) This work is licensed under Creative Commons Attribution Non Commercial 4.0 International License. The full terms of the License are available at http://creativecommons.org/licenses/by-nc/4.0 ing. In an attempt to yield empirical data supporting the need for such initiatives, the authors have conducted analysis on the publication and authorship patterns of female researchers in ICAD conferences.

Over the past few years, several similar papers have been published about other related communities, such as the Audio Engineering Society (AES), the International Computer Music Association (ICMA) [2], and the Conferences of the International Society for Music Information Retrieval (ISMIR) [3]. Even though crossstudy comparisons are not feasible due to differences in methodology and data analysis, each publication offers a valuable perspective on the topic. Hopefully, this increase in the number of studies revealing the under-representation of women scientists and artists in audio and music-related research-fields, will stimulate people's interest in this issue and lead to the necessary actions to resolve the situation.

\section{RELATED WORK}

In the majority of technical fields the number of female researchers and scholars is significantly smaller than that of their male colleagues. A report from the American Association of University Women states that women account for $17 \%$ of the high school students who take advanced placement exams in computer science, and for $28 \%$ of the undergraduate degrees awarded on related disciplines. Similar to computer science, in the fields electronic and computer music both the aesthetic path of music composition and the technical aspects of audio engineering and recording have been dominated by male researchers.

The early literature on $20^{t h}$ century music undermined women's achievements in composition and performance [4]. Early music composition books referenced one to two female composers. It was not until the mid ' 90 s that female electronic music composers such as Pauline Oliveros and Kaija Saariaho started to be included in textbooks, and gender-biased computer music advertising to be criticized [5]. An attempt to shed light on this situation was made by Mathew et al. [2], who attributed women's under-representation in fields related to computer music and audio to a) social and environmental factors [6], b) lack of role models [7], and c) issues related to work-life balance [8].

Women's under-representation extends beyond education, artistic creation, and engineering to scientific research. This was demonstrated in a study by Sugimoto et al.[9] who investigated the percentage of female authors in 5,483,841 articles published between 2008 and 2012 in the Web of Science, an online database 
of 27 million publications. Results indicated that only $30 \%$ of the authors were female. Hence, the gender gap in scientific research publications appears to be prominent in a much broader range of disciplines. In certain cases, this bias seems to affect how women's work is valued within a scientific community. A study of citation patterns based on gender in the field of International Relations [10] reported that papers authored by women were cited significantly less than those written by men. Nevertheless, it is worth mentioning here that a similar study on the scientific works of the audiorelated ISMIR community revealed no such tendencies [11].

Previously published work on the representation of women scientists and artists in music and audio-related fields has found the rates of female members to be $<20 \%$ in the International Computer Music Association (ICMA) and $<10 \%$ in the Audio Engineering Society (AES) [2]. Similar analysis on the representation of female authors in the proceedings of the Conferences of the International Society for Music Information Retrieval (ISMIR) estimated the participation of female authors to vary between $10 \%$ and $20 \%[3,11]$. This paper will present an overview of the representation of female scientists and artists in the published proceedings of all ICAD conferences since 1994.

\section{METHODOLOGY}

\subsection{Data collection and standardization}

In the absence of either a membership mechanism or publicly available records of ICAD conference attendees, the investigation of the representation of women in the International Community of Auditory Displays was based exclusively on the infometrics of authors with published papers in ICAD conferences. ICAD proceedings, which are hosted by the SMARTech Repository service of the Georgia Tech Library, are publicly available under a Creative Commons Attribution 4.0 International License.

Records of papers and authors for all available conferenceyears between 1994 and 2016 were extracted from the SMARTech website and stored into spreadsheets. Data extraction was followed by a standardization procedure during which authors were cataloged in the following format: ["Last name", "First name" "Initials of middle names (if any)"]. For cases where only the initials of first names were provided, full names were manually retrieved through the search of public records on the Internet. Following that, the authors' gender was manually determined based on their first names. Additional information on the number of authors per publication and their affiliation was also logged.

\subsection{Limitations}

The authors acknowledge that the selected methodology for the study of women's representation in the International Community for Auditory Display suffers from certain limitations. First, a study of the representation of female authors in ICAD publications cannot fully reflect the involvement of women in the conferences, where their role could be that of an attendee, conference staff, or organizer, nor their involvement in the community in general. Nevertheless, in the absence of an official ICAD membership mechanism, published ICAD proceedings are the only reliable and publicly available source of information, to date.

In addition, the procedure followed to determine the authors' gender based on their first-names is error-prone for various rea-

\footnotetext{
${ }^{0} \mathrm{https} / / /$ smartech.gatech.edu/
}

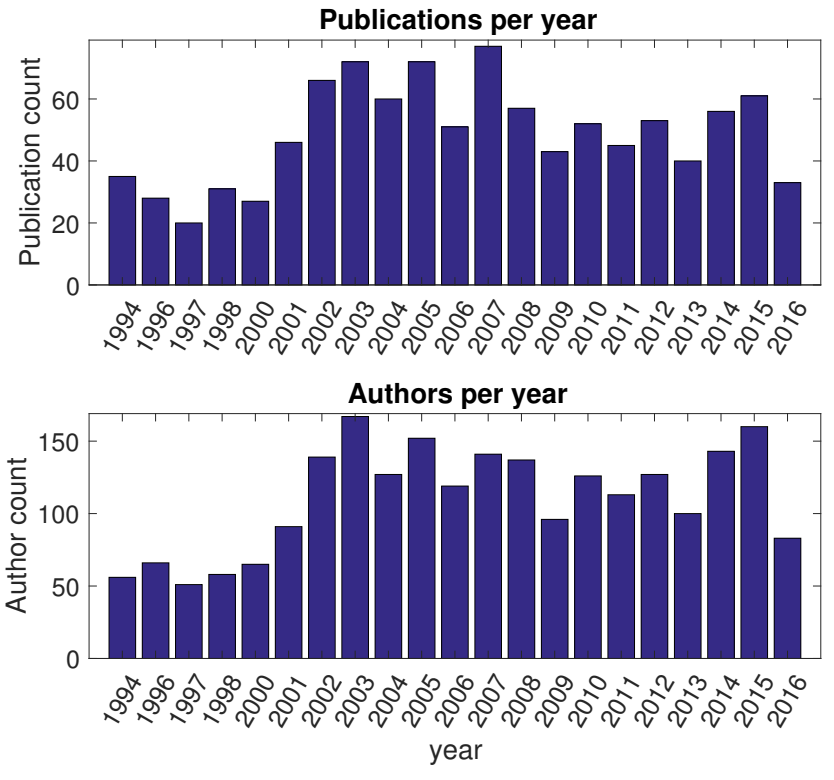

Figure 1: Evolution of the number of published ICAD papers per conference year (top). Evolution of the number unique authors per conference year (bottom)

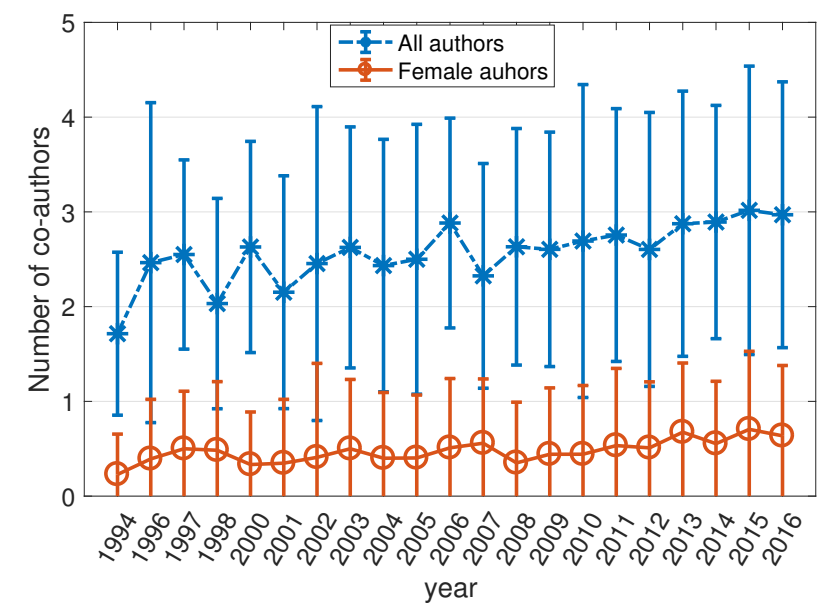

Figure 2: Evolution of the average number co-authors vs the average number of female authors per paper and conference year. Error bars mark the range of \pm 1 Standard Deviation (std) from the mean.

sons. First-names are not always gender specific across all languages. For cases where it was not possible to determine one's gender based on their name, a search on the Internet was performed using the author's full name and affiliation and a gender label was assigned based on photographs and / or online bios. Moreover, certain names can be exclusively attributed to a single gender in one language but be gender neutral or attributed to the opposite gender in other languages. Even though gender labels were assigned with caution and, to the extend possible, in accordance with the author's nationality, it is possible that some of the data have been mislabelled. Finally, this work uses a simplified binary gender distinction to refer to all ICAD authors. It is possible that some 


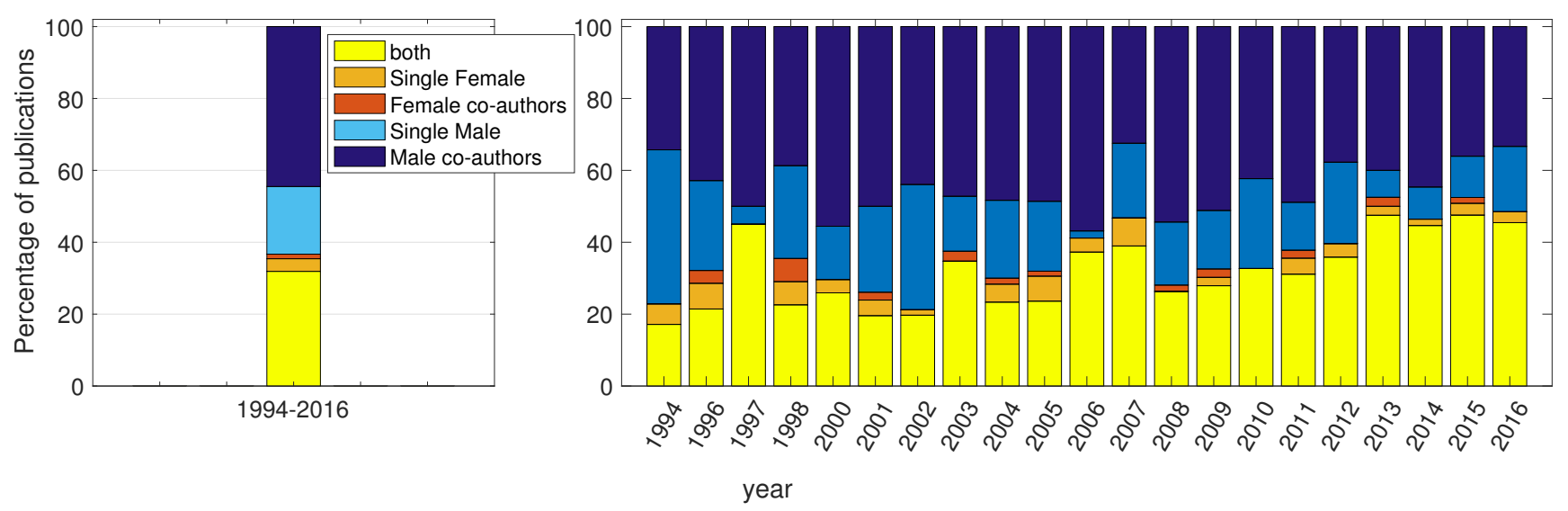

Figure 3: Percentage of publications with a) both male and female co-authors (yellow); b) single female author (light orange); c) only female co-authors (dark orange); d) single male author (light blue); e) only male co-authors (dark blue). Analysis is shown across all conference years (left) and per conference year (right)

authors either do not embrace this distinction or self-identify with the opposite gender to the one customary attributed to their first names. Inevitably, such cases cannot be accurately represented in the current data.

\section{RESULTS}

\subsection{Female co-authorship in ICAD publications}

Between 1994 and 2016 the International Community for Auditory Display has organized 21 conferences around the globe, 8 of which were held in the United States, 9 in Europe, 2 in Australia, 1 in Canada, and 1 in Asia. ICAD is a single track conference with an average of 48.8 papers $(\min =20, \max =77$, std $=16.16)$ and 110 authors $(\min =51, \max =167, \mathrm{std}=36.4)$ per year. As can be seen in Figure 1, both the number of publications (top) and the corresponding number of unique authors (bottom) have the tendency to grow. This increase has been clearer since 2001, shortly after the conference started to travel globally.

Over the years, in addition to the growing number of publications, a shift towards more collaborative projects seems to have taken place. More specifically, as can be seen in Figure 2 while for the first conference in 1994 the average number of co-authors per publication was $1.7(\mathrm{std}=0.86)$, by 2016 it reached $2.9(\mathrm{std}=$ $1.4)$, with a maximum of $3(\mathrm{std}=1.5)$ in 2015 . Nevertheless, this trend does not fully reflect the participation of female authors in ICAD publications. In 1994 the average number of female authors per paper was as low as $0.2($ std $=0.43)$. Even though this number has increased by a factor of 3.5 over the course of 21 conferences (Figure 2), reaching a maximum of $0.7(\mathrm{std}=0.82)$ in 2015 , it still remains at very low levels.

Figure 3 (left) shows the combined co-authorship analysis across all ICAD conferences since 1994. Overall, only 36.7\% of all papers include at least 1 female co-author. In more detail, $63.3 \%$ of them were written exclusively by male collaborators, $31.9 \%$ were the result of a collaboration between male and female researchers, and only $4.8 \%$ were authored exclusively by female researchers. It is worth pointing out that $30 \%$ of the male authored papers were single-author publications, while for female authored publications that percentage was as high as $70 \%$. In order to explore the evolution in the representation of female authors in ICAD publications, a co-authorship analysis per conference year was also performed. As can be seen in Figure 3 (right), with the exception of the 1997 and 2007 conferences, when the percentage of publications with at least one female author reached $45 \%$ and $46.8 \%$, it was not until 2013 that more that half of the papers $(52.5 \%)$ included female researchers.

In general, the number of published ICAD papers, co-authored by at least 1 female researcher, seems to be consistently increasing. The conference years with the lowest female representation were $1994(22.9 \%)$ and $2002(22.2 \%)$, and those with the highest were $2013(52.5 \%)$ and $2015(52.4 \%)$. The percentage of papers written exclussively by female authors does not seem to follow the same trend. The highest percentages of such papers, including singleauthor publications, appeared during the earlier ICAD years, prior to 2008 , reaching a maximum of $13 \%$ in 1998 . Since 2008 , the corresponding percentages have varied between $1.5 \%$ and $6.6 \%$. Another interesting observation is that the number of publications authored by a single female author is consistently larger than that of papers written by a group of female-only scientists. This observation implies, that female ICAD authors are more likely to publish as part of a mixed-gender author group or as single researchers, than forming female-exclusive groups. In contrast to that behavior, for male authors the formation of single-gender author-groups remains a very popular option throughout the ICAD conference years.

\subsection{Female representation in ICAD publications}

A more detailed analysis of female co-authorship in ICAD followed, looking at the percentages of papers with a) a leading female author, b) a last female author, c) both leading and last female authors, d) female co-authors and e) single authored papers. The choice to focus on leading and last authors is not coincidental, but rather driven by a customary academic convention, according to which first authors are usually scholars and last authors supervising researchers. Both roles are of particular interest, as they reflect the representation of prospective and established researchers within the ICAD community. We acknowledge that while this convention is well established within many scientific research do- 


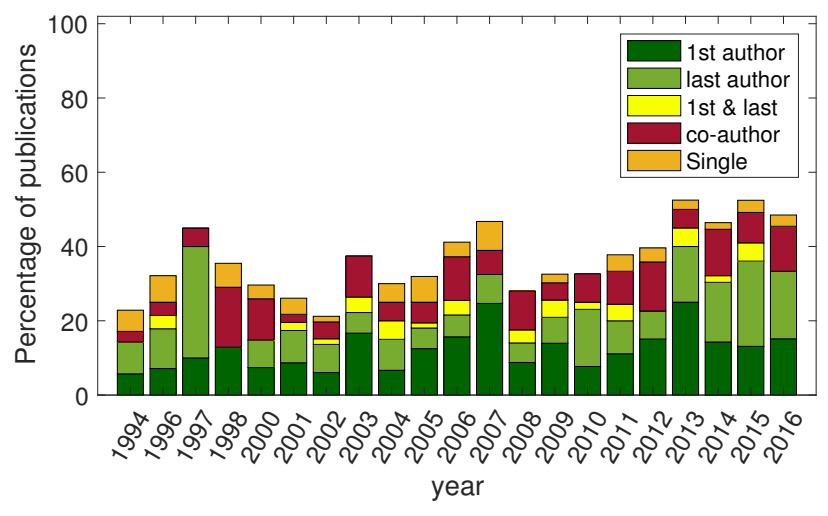

Figure 5: Analysis of publications including at least 1 female coauthor, per conference year. In dark green: the percentage of papers with a leading female author; in light green: the percentage of papers with a last female author; in yellow: the percentage of papers with both leading and last female authors; in red: the percentage of papers with female co-authors in other roles; in orange: the percentage of single-author papers.

mains, it may not hold true for the humanities and the arts. Nevertheless, it still represents a portion of scholar work within the broad field of auditory displays, and is therefore worth exploring.

As can be seen in Figure 5, during the early conferences, the percentage of last female authors was considerably larger than that of leading authors. In the following years this relationship was occasionally inverted and approached an equilibrium in the last 5 conferences. Interestingly enough, throughout the history of ICAD conferences the percentage of publications with at least 1 female co-author was consistently smaller than the sum of percentages of papers with a leading and / or last female author. These findings imply that, even though in the recent years there exists an equivalent percentage of published ICAD papers with female scientists in leading or supervising roles, it is much less likely to encounter papers with female co-authors, for which the aforementioned research roles have been undertaken by male researchers. It is also worth pointing that the percentage of papers having both leading and last female authors was, with very few exceptions, smaller than any other of the aforementioned subgroups including single authored papers, fact which further supports the previously discussed observation that female researchers in the ICAD community are more likely to collaborate with other male colleagues than form female-exclusive research teams (see also Figure 3).

Up to this point, the data analysis focused on the number of published ICAD papers and the representation of female coauthors per publication and year. This perspective, even though informative as it reflects the growth and evolution of scientific collaborations within the ICAD community, does not really address the focal point of this paper which is the representation of women in ICAD conferences. An increase in the number of papers coauthored by female scientists might as well be the result of multiple concurrent collaborations of the same few female authors, rather than of an actual increase in the number of authors involved in the community. As a result, a different data analysis method was explored evaluating the percentage of female authors across all ICAD conferences.

Out of the 1520 unique authors who have published in ICAD conferences since 1994 only 20\% were female (Figure 4-left). An analysis on their representation per conference year (Figure 4 (right)) revealed that levels have slightly increased but, in general, remain very low, ranging between $10.8 \%$ and $29 \%$ (mean $=18.7 \%$, std $=4 \%$ ). Such findings imply that the previously discussed increase in the participation of female authors in ICAD publications (Section 4.1) is related to the general growth of the annual conferences, and does not reflect an increase in the actual number of female scientist and artists who have chosen to publish with ICAD.

The 304 identified female authors who have published in ICAD conferences were affiliated with 188 institutions and / or companies from 25 countries worldwide. Table 1 shows the Top10 countries ranked according to the number of female authors they have hosted. As can be seen, the vast majority of female authors $(41.3 \%)$ have been associated with at least one institution in the USA at some point in their career. Other countries with relatively large female ICAD populations are: the UK, France, Canada, Austria, Germany, Australia, Finland, Japan, and Italy. Table 2 complements this information with a list of Institutions ranked according to the number of female ICAD authors they have hosted. Even though further discussion on this topic is not possible
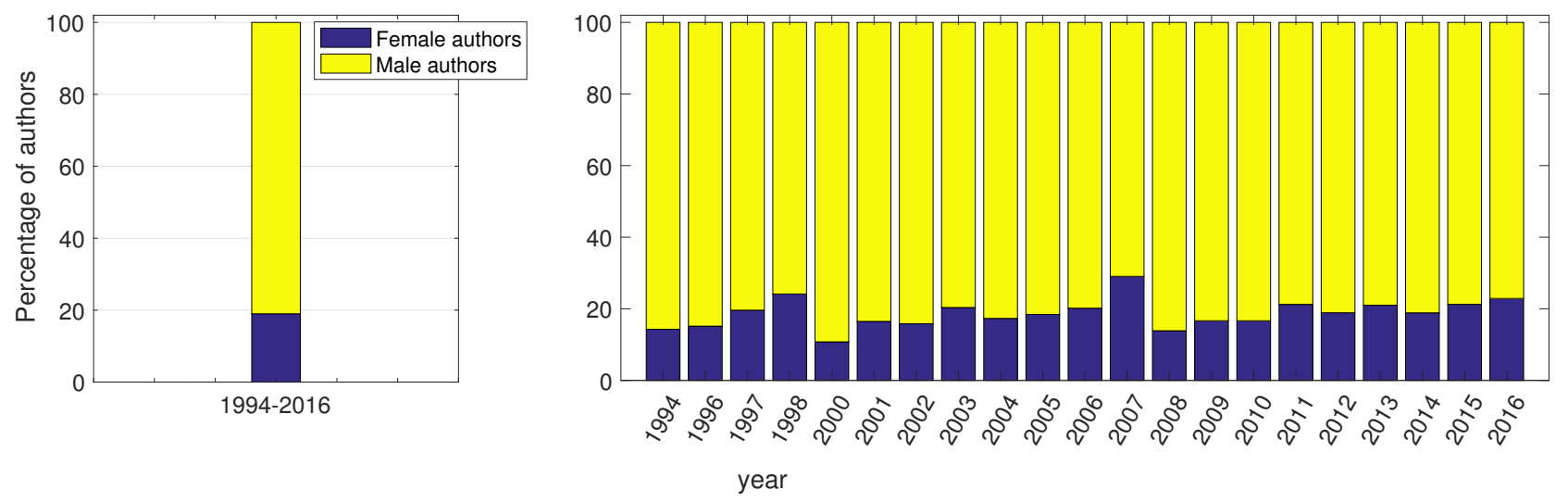

Figure 4: Percentage of unique female (blue) and male (yellow) authors, across all conference years (left), per conference year (right). 
Table 1: Countries with the largest percepntage of female ICAD authors

\begin{tabular}{|r|l|c|}
\hline Index & Country & \% of Female Authors \\
\hline \hline 1 & USA & 41.3 \\
2 & UK & 14.8 \\
3 & France & 7.4 \\
4 & Canada & 5.5 \\
5 & Austria \& Germany & 4.2 \\
7 & Australia & 3.5 \\
8 & Finland \& Japan & 3.2 \\
10 & Italy & 2.9 \\
$11-25$ & Others & 9.1 \\
\hline
\end{tabular}

Table 2: Institution ranking according to the number of affiliated female ICAD authors.

\begin{tabular}{|l|c|}
\hline Institution & \# of Female Authors \\
\hline \hline Georgia Institute of Technology & 9 \\
University of York & 9 \\
CNRS & 7 \\
Michigan Technological University & 7 \\
University of British Columbia & 7 \\
University of Michigan & 7 \\
University of Glasgow & 6 \\
Boston University & 5 \\
IMASSA & 5 \\
Universita di Salerno & 5 \\
\hline
\end{tabular}

unless a similar analysis per country and institution is performed for all male ICAD authors, these two tables are included for future reference and discussion.

\subsection{Returning ICAD community members}

The very small fluctuations in the representation of male and female authors in ICAD conferences, discussed in Section 4.2, give rise to the following two questions: a) is the International Community for Auditory Display dominated by an increased number of returning researchers and artists who keep publishing their work in ICAD conferences, and b) does there exist a strong community of female researchers and artists who have chosen to follow and publish their work in ICAD conferences? In an attempt to address these points, distributions of recurrent male and female authors were created and compared using a two-sample unequal variance t-test. Results showed no significant difference between the two distributions $(t(553)=-1.16, p=0.42)$ at the default $5 \%$ significance level, and a Cohen's d effect size of $d=0,07$, suggesting that both genders' "loyalty" to ICAD conferences is similar.

In order to assist visibility, the aforementioned distributions were converted into percentages and divided into the following 3 subgroups: percentage of male and female authors who published in the conference a) once, b) up to three times, and c) more than four times. As can be seen in Figure 6, 81\% of all female and $77.1 \%$ of all male authors have published their work in a single ICAD conference, while $12.8 .4 \%$ and $16.3 \%$ in up to 3 , and $6.3 \%$ and $6.6 \%$ in 4 or more conferences, respectively. An investigation of potential reasons for the significantly large number of authors

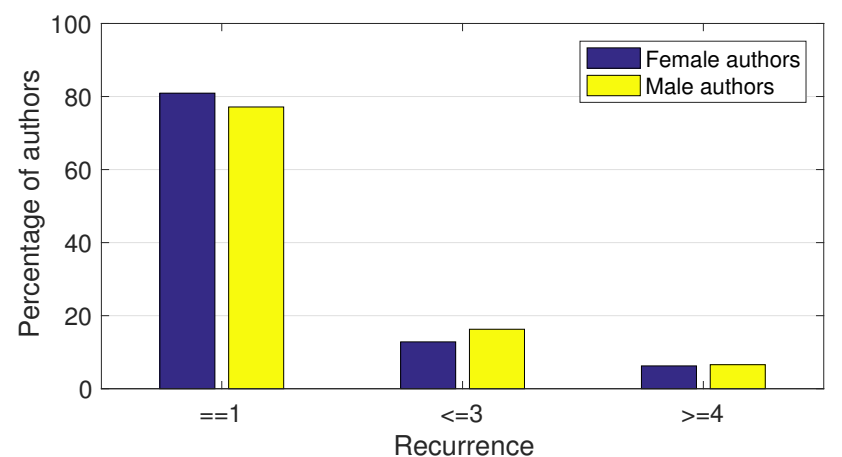

Figure 6: Comparison of the recurrence of female (blue) and male (yellow) authors in ICAD conferences over the years.

who have chosen to publish in a single ICAD conference, and how does it compare with that of other long-lasting conferences is beyond the scope of this paper, and should be revisited separately. Nevertheless, what remains of interest is the lack of evidence of any gender bias on ICAD "loyalty". Even though the absolute numbers of male and female published authors are significantly distinct, the choice of whether or not to follow this conference in its journey over the years does not seem to be affected by gender.

\section{DISCUSSION AND FUTURE WORK}

Over the past few years, there has been an increase in the number of publications studying women's involvement in musicproduction, composition, and audio-related research fields. To no surprise, albeit somewhat disappointingly, women's involvement in these artistic and scientific areas as been found to be alarmingly small. Several factors leading to this situation have been previously proposed, such as a) social expectations [6], b) lack of rolemodels [7], and c) issues related to work-life balance [8]. Most related literature concludes that, in order to encourage young female researchers and artists to be involved in music and audio-related fields, we need to study the achievements of experienced female researchers, and learn from their career paths. With this work, the authors wish to contribute to the understanding of women's representation in music and audio technology fields, by focusing on a study of female author participation in the conferences of the International Community for Auditory Display.

A simple gender analysis of all ICAD authors since 1994 revealed that women account for less than $20 \%$ of the published researchers across almost every conference. This percentage, even though low, is directly comparable to those of other related communities, such as the International Computer Music Association (ICMA) [2], and the International Society for Music Information Retrieval (ISMIR) [3] and almost double the percentage of female Audio Engineering Society (AES) members over the past decade, which is as low as $10 \%$. The significant drop in the number of women affiliated with the AES community can possibly be attributed to its more technically oriented scope, which traditionally focused on audio signal processing and engineering.

While a more in-depth comparison between the studies of the aforementioned communities and the results of this paper cannot take place due to differences in data collection and analysis, their 
statistics still serve as a very valuable comparison for the existing gender-related tendencies in the fields of music and audio research. The authors acknowledge that the study of female authors in ICAD conferences is not equivalent to a study of the representation of women in the International Community for Auditory Displays. Active involvement in a community can be manifested in various ways beyond scientific publications, such as conference attendance, conference organization, or even through the involvement with the community's Board of Directors. Future work will involve the collection and analysis of additional data related to the conference organization and attendance in an attempt to get a more complete view of women's involvement in the community. Additional points of interest include a study of the effect of geographic location on the infometrics of ICAD conferences, as well as the investigation of choice of research topics undertaken by male and female researchers.

\section{REFERENCES}

[1] C. Frauenberger and T. Stockman, "Auditory display designan investigation of a design pattern approach," International Journal of Human-Computer Studies, vol. 67, no. 11, pp. 907-922, 2009.

[2] M. Mathew, J. Grossman, and A. Andreopoulou, "Women in Audio: contributions and challenges in music technology and production," in 141st Audio Engineering Society Convention, Los Angeles, USA, 2016, pp. 1-10.

[3] K. Choi, J. H. Lee, A. Laplante, Y. Hao, S. J. Cunningham, J. S. Downie, and U. Washington, "Wimir : an Informetric Study on Women Authors in Ismir," Proc. 17th International Society for Music Information Retrieval Conference, pp. 765-771, 2016.

[4] C. E. Seashore, "Why no great women composers?" Music Educators Journal, vol. 26, no. 5, pp. 21-88, 1940.

[5] A. McCartney, "Inventing images: constructing and contesting gender in thinking about electroacoustic music," Leonardo Music Journal, vol. 5, no. 1, pp. 57-66, 1995.

[6] _ _ "In and out of the sound studio," Organised Sound, vol. 8, no. 01, pp. 89-96, 2003.

[7] K. De Welde, S. Laursen, and H. Thiry, "Women in science, technology, engineering and math (stem)," 2007.

[8] J. C. Williams, "The 5 biases pushing women out of stem," Harvard Business Review, 2015.

[9] C. R. Sugimoto, V. Lariviere, C. Ni, Y. Gingras, B. Cronin, et al., "Global gender disparities in science," Nature, vol. 504, no. 7479, pp. 211-213, 2013.

[10] D. Maliniak, R. Powers, and B. F. Walter, "The gender citation gap in international relations," International Organization, vol. 67, no. 04, pp. 889-922, 2013.

[11] X. Hu, K. Choi, J. H. Lee, A. Laplante, Y. Hao, S. J. Cunningham, and J. S. Downie, "Wimir: An informetric study on women authors in ismir," in Proceedings of the 17th International Conference on Music Information Retrieval (ISMIR), 2016. 